\title{
A library of shortcuts for faster image making with PyMOL
}

\author{
Blaine Mooers
}

975 NE $10^{\text {th }}$ St., BRC 466, Department of Biochemistry and Molecular Biology, University of Oklahoma Health Sciences Center, Oklahoma City, OK 73104, USA, blaine-mooers@ouhsc.edu

PyMOL is often used to make publication quality images of biological molecular structures. We developed a set of over 180 Python functions that extend and ease the use of PyMOL. These functions are loaded automatically on startup. A user runs a function by entering its name on the command line. The names of the functions are 2-4 characters long, so they are fast to enter. The builtin help function displays the documentation of a function in the GUI. The 'SC' function refreshes the list of the shortcuts in the command history window. These functions are also called shortcuts and are similar to 'aliases' [1].

We clustered the shortcuts by functionality for faster look-up. One cluster generates molecular representations and visual effects that are not available in PyMOL (e.g., ambient occlusion). A second group opens text editors that have snippet libraries of PyMOL code. A third group saves files with time stamps in the file name. The unique filenames avoid overwriting incrementally-developed script and session files. A fourth group submits search terms to PubMed and elsewhere in the user's web browser. A fifth group enables editing of png files without leaving PyMOL. The user can work longer inside PyMOL without interruption when using the shortcuts.

The shortcuts should save the average PyMOL user many hours of work per year. The shortcuts are available at http:github.com/MooersLab/pymolshortcuts.

References

[1] Mooers, B. (2016) Protein Sci., 25, 1873-82. 